\title{
Aplicación de primera derivada en solidificación de aleaciones
}

\author{
Belmares, Sergio \\ Aplicación de primera derivada en solidificación de aleaciones \\ CIENCIA ergo-sum, vol. 25, núm. 1, 2018|e7 \\ Universidad Autónoma del Estado de México, México
}

Esta obra está bajo una Licencia Creative Commons Atribución-NoComercial-SinDerivar 4.0 Internacional.

Belmares, S. (2018). Aplicación de primera derivada en solidificación de aleaciones. CIENCIA ergo-sum, 25(1). Disponible en: http://cienciaergosum.uaemex.mx/article/view/9259 


\section{Aplicación de primera derivada en solidificación de aleaciones}

First derivative implementation in solidification alloys

Sergio Belmares

Universidad Autónoma de Nuevo León, México

Recepción: 25 de octubre de 2016

sergiobelmares@msn.com

\section{Resumen:}

Se realiza una aplicación numérica de la derivada. También, se presenta el algoritmo de un programa basado en el método de mínimos cuadrados. El programa es utilizado para el cálculo experimental de la temperatura de fusión de una aleación de aluminio, de alrededor de $660{ }^{\circ} \mathrm{C}$, dando un error relativo del $0.08 \%$. Asimismo, se utiliza esta derivada para evaluar su efectividad en funciones periódicas y continuas a trozos; en todos los resultados se obtiene un coeficiente de correlación de 1.0. El trabajo está enfocado a estudiantes de licenciatura en cálculo diferencial e investigadores que requieran de la derivada con análisis numérico.

Palabras Clave: derivada, mínimos cuadrados, análisis térmico, análisis numérico.

\section{Abstract:}

In this paper a numerical application of the derivative is performed, the algorithm based on the least squares method program is presented. The program is used for calculation of experimental melting temperature of an aluminum alloy, of about $660^{\circ} \mathrm{C}$, giving a relative error of $0.08 \%$. This derivative is also used to evaluate its effectiveness in periodic and piecewise continuous functions, a correlation coefficient of 1.0 is obtained in all results. The work presented here is focused to undergraduate students in differential calculus and researchers that require the derivative with numerical analysis.

KEYWORDS: derivative, least squares, thermal analysis, numerical analysis.

\section{INTRODUCCIÓN}

La primera aplicación importante de la derivada de una función la realizó Fermat (1891), quien la llevó a cabo en 1629, pero nunca tuvo la iniciativa de publicar un artículo en su vida. Su trabajo se publicó hasta 1679, después de su muerte (Biacino, 2014). Con este antecedente, dentro de este artículo se encuentra el método de máximos y mínimos de funciones algebraicas; sin embargo, la formulación de la derivada y del cálculo fue hecha por Leibniz (1646-1716) y Sir Isaac Newton (1642-1727) (Leithold, 2014), ambos trabajando de manera independiente. Hasta la actualidad hay un sinnúmero de áreas en donde se utiliza la derivada; algunas de las más importantes son las matemáticas, la física, la biología y la economía (Singh et al., 2014).

Algunos problemas en física e ingeniería requieren de diferenciación e integración con datos discretos obtenidos de experimentos, en donde se hace uso de tarjetas de adquisición de datos (Martínez et al., 2016). La manera de tratar estos datos discretos es por medio de un análisis numérico (Chapra y Canale, 2007). En el caso de diferenciación numérica se utilizan más de dos datos para dar un resultado más exacto de la derivada, algunos métodos numéricos como el de Richardson utilizan cinco datos por cada cálculo de la derivada más una estimación centrada(Chapra y Canale, 2007). El resultado final es equivalente a ajustar un polinomio de grado superior a los datos y después evaluar las derivadas con diferencias divididas centradas. Este artículo emplea un método que calcula la derivada de datos discretos y utiliza también el método de mínimos cuadrados (Baird, 1991). Se escogió este método por calcular la pendiente de una recta que más se ajusta a datos experimentales de registro de temperatura en solidificación de aleaciones. Se asume que la pendiente de esta línea recta se acerca a la primera derivada de la curva de enfriamiento. Las observaciones experimentales de este trabajo dan como resultado un mejor ajuste con seis datos por cada cálculo. 


\section{ApliCACIÓN DE LA DERIVADA EN SOLIDIFICACIÓN DE ALEACIONES}

En ciencia de materiales se aplica la derivada en análisis térmico (Narayanan et al., 1994). Primero se eleva la temperatura en un metal por encima de su punto de fusión hasta su estado líquido, después se enfría y la temperatura se registra con un termopar y una tarjeta de adquisición de datos. Con este registro, temperatura en función del tiempo, se obtiene una curva denominada curva de enfriamiento, en la cual la temperatura baja primero de manera lineal al pasar el tiempo, hasta que aparece una fase, y en la curva se presenta una meseta; es decir, cada inicio de meseta representa las temperaturas de precipitación de cada fase.

Cuando en la curva de enfriamiento se detecta con claridad la formación de una meseta, en el inicio de ésta se obtiene directamente la temperatura de precipitación de la fase; sin embargo, cuando en la curva de enfriamiento no se detecta el inicio de la meseta, o existe recalescencia, se hace uso de la primera derivada. Cuando la primera derivada es cero, se dice que es un punto crítico (Fermat, 1891; Leithold, 2014), un máximo o un mínimo que en solidificación se interpreta como algo crítico, esto es, el inicio de precipitación de una nueva fase. En resumen, de la curva de enfriamiento se puede obtener la primera derivada y cuando ésta es cero se obtiene el inicio de precipitación de fase. Este resultado es importante en solidificación porque la cinética de precipitación de fases dicta las propiedades de la aleación (Narayanan et al., 1994). Como ya se mencionó, para el cálculo de la primera derivada se utilizó el método de mínimos cuadrados.

\section{MÉtodo de mínimos cuadrados}

Si trazamos la línea entre dos puntos cualesquiera en un plano A y B, y consideramos los intervalos verticales entre los puntos experimentales $\left(P_{i}\right)$ y los puntos de la línea recta $\left(O_{i}\right)$, entonces se define la mejor línea $\mathrm{AB}$ como aquella que minimiza la suma de los cuadrados de las desviaciones $P_{i} O_{i} \quad$ (Baird, 1991).

Expresemos ahora el principio de mínimos cuadrados en forma matemática. Definimos que la mejor línea es aquella que lleva a su valor mínimo la suma:

$$
\sum\left(P_{i} O_{i}\right)
$$

Y deseamos obtener los parámetros, pendiente $m$ y ordenada al origen $b$, de esa mejor línea.

Sea la ecuación de la mejor línea (2):

$$
y=m x+b
$$

La magnitud de la desviación $P_{i} O_{i}$ es el intervalo entre un cierto valor medido $y_{i}$ y el valor de $y$ en ese punto, para el valor de $x$. Este valor $y$ se puede calcular a partir del valor correspondiente de $x$ como $m x_{i}+b$, de manera que si nombramos $\delta y_{i}$ a cada diferencia, tenemos:

$$
\delta y_{i}=y_{i}-\left(m x_{i}+\mathrm{b}\right)
$$

El criterio de mínimos cuadrados nos permite obtener los valores de $m$ y $b$. A partir de las ecuaciones (1) y (3) se establece la condición (4):

$$
\sum\left[y_{i}-\left(m x_{i}+b\right)\right]^{2}=\text { mínimo }
$$


Y reescribimos la ecuación anterior como:

$$
\sum\left[y_{i}-\left(m x_{i}+b\right)\right]^{2}=M
$$

Desarrollando la ecuación (5), obtenemos la ecuación (6):

$$
\mathrm{M}=\sum\left(y_{i}^{2}+m^{2} x_{i}^{2}+b^{2}+2 m x_{i} b-2 m x_{i} y_{i}-2 y_{i} b\right)
$$

Desarrollando aún más, tenemos la ecuación (7):

$$
M=\sum y_{i}^{2}+m^{2} \sum x_{i}^{2}+N b^{2}-2 m b \sum x_{i}-2 m \sum x_{i} y_{i}-2 b \sum y_{i}
$$

Luego, la condición para que sea un mínimo es:

$$
\frac{\partial M}{\partial m}=0 \mathrm{y} \frac{\partial M}{\partial b}=0
$$

La primera condición de la ecuación (8) aplicada a la ecuación (7) da como resultado (9):

$$
2 m \sum x_{i}^{2}+2 b \sum x_{i}-2 \sum\left(x_{i} y_{i}\right)=0
$$

Y la segunda condición de la ecuación (8) aplicada a la ecuación (7) da como resultado (10):

$$
2 \mathrm{Nb}+2 \mathrm{~m} \sum \mathrm{x}_{\mathrm{i}}-2 \sum \mathrm{y}_{\mathrm{i}}=0
$$

Con las dos ecuaciones anteriores se realiza resolución simultánea y se obtienen (11) y (12):

$$
\begin{gathered}
m=\frac{N \sum\left(x_{i} y_{i}\right)-\sum x_{i} \sum y_{i}}{N \sum x_{i}^{2}-\left(\sum x_{i}\right)^{2}} \\
b=\frac{\sum x_{i}^{2} \sum y_{i}-\sum x_{i} \sum\left(x_{i} y_{i}\right)}{N \sum x_{i}^{2}-\left(\sum x_{i}\right)^{2}}
\end{gathered}
$$

También, se pueden hacer cálculos de la desviación estándar de la pendiente y la ordenada, pero por ahora lo que más interesa es encontrar la correlación de datos del cálculo con mínimos cuadrados y las funciones reales, como más tarde se analizará. 


\section{AlgoritMo DEL PRograma de MíNImos CuAdrados}

Para implementar el método de mínimos cuadrados y tratar los datos discretos experimentales se utilizó un programa con SigmaPlot ${ }^{\circ}$, que es una hoja de cálculo que permite hacer programas sobre los datos en sus columnas y mostrar los resultados en otra u otras columnas diferentes. El programa fue hecho en código de SigmaPlot ${ }^{\circledR}$ y calcula los valores de $m$ de la ecuación (11) cada seis datos. Como ya se mencionó, se asume que los resultados de $m$ se acercan a la primera derivada.

En este algoritmo se utilizan los datos de entrada como sigue: en la columna 1 los datos correspondientes a $x_{i}$ de la ecuación 11, en la columna 2 los datos de $y_{i}$. Y se encuentran los datos de salida como sigue: en la columna 30 los resultados de $x_{i}^{2}$, en la columna 31 los resultados de $x_{i} y_{i}$ y finalmente en la columna 10 los resultados de $m$. Este algoritmo fue diseñado originalmente para tratar datos experimentales de curvas de enfriamiento, pero se puede implementar para cualquier tipo de curva construida con datos discretos. Si se requiere obtener la derivada de una función continua, simplemente se tabulan resultados de la función con diferentes datos de la variable independiente .

La adquisición de los datos para las columnas 1 y 2 del algoritmo anterior se realizó con la experimentación.

Para ejecutar el programa, primero se abre SigmaPlot ${ }^{\circ}$ en la sección de datos, después se dirige a la pantalla análisis y se puede copiar, guardar y ejecutar el siguiente algoritmo en la casilla Transform, y luego Userdefined. El algoritmo que se implementó se encuentra en el Anexo.

\section{EXPERIMENTACIÓN}

La composición en peso de la aleación utilizada de este trabajo se presenta en el cuadro 1. Para el análisis térmico parte del metal fue colado en el molde cilíndrico de grafito representado en la figura 1, similar al molde utilizado por Backerud et al. (1990). Antes de vaciar el metal, el molde de grafito fue calentado con la ayuda de un horno de resistencias a $750{ }^{\circ} \mathrm{C}$. Una vez vertido el metal dentro del molde, se mantuvo a $750{ }^{\circ} \mathrm{C}$ por espacio de dos minutos para posteriormente retirar el horno e iniciar el enfriamiento de la copa mediante la inyección de aire controlada. Se empleó un termopar tipo K limit of error. La señal del termopar fue digitalizada mediante una tarjeta de adquisición de datos Keithley DAS-TC y de allí se enviaron los datos a una computadora de escritorio. Este registro da como resultado la curva de enfriamiento experimental buscada.

\section{CUADRO 1}

Composición química de la aleación estudiada

\begin{tabular}{|lcccccc}
\hline \multicolumn{7}{c}{ Elemento (Wt Pct) } \\
\hline Aleación & $\mathrm{Al}$ & $\mathrm{Zn}$ & $\mathrm{Cu}$ & $\mathrm{Si}$ & $\mathrm{Fe}$ & $\mathrm{Mg}$ \\
Porcentaje & 99.82 & --- & --- & 0.05 & 0.13 & --- \\
& & & & & & \\
\hline
\end{tabular}

Fuente: elaboración propia. 


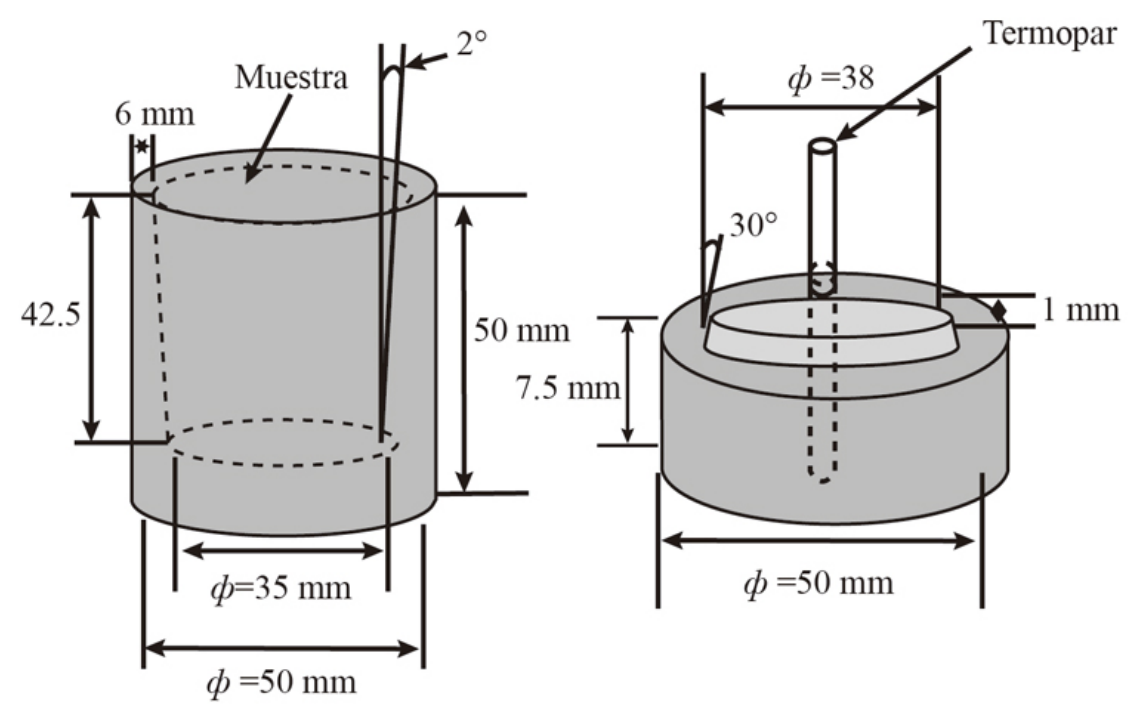

FIGURA 1

Molde de grafito y tapa para muestras de análisis térmico Fuente: elaboración propia.

Hasta aquí cabe la siguiente pregunta: ¿se podría esperar a futuro hacer la experimentación para otras aleaciones y tener buenos resultados de aplicación de derivada? La aleación del cuadro 1 se escogió debido a que en las condiciones del experimento tenemos velocidades de enfriamiento altas del orden de $12{ }^{\circ} \mathrm{C} /$ s. Siendo que en otras aleaciones donde se tienen más elementos como el $\mathrm{Mn}, \mathrm{Mg}$ y $\mathrm{Cu}$, con composición comercial como la aleación Al319, se obtienen velocidades de enfriamiento mucho más bajas del orden de $2.5^{\circ} \mathrm{C} / \mathrm{s}$ (Narayanan et al., 1994). Si el algoritmo del programa de mínimos cuadrados da buenos resultados con la aleación del cuadro 1 , entonces en un futuro este algoritmo se podría utilizar fácilmente para cualquier otra aleación puesto que se tendrían velocidades de enfriamiento más bajas y así se obtendrían cálculos más certeros.

\section{Resultados y DiscusióN}

La temperatura de fusión de aluminio puro es de $660^{\circ} \mathrm{C}$, que es una temperatura bien conocida (Backerud et al., 1990). Para encontrar experimentalmente la temperatura de fusión de una aleación de aluminio, se utilizó y evaluó la curva de enfriamiento de la aleación del cuadro 1 y el algoritmo del programa de mínimos cuadrados. Después, se discuten los datos del error relativo de esta medición y finalmente la confiabilidad del algoritmo del programa que utiliza el método de mínimos cuadrados descrito.

En la gráfica 1 se muestra la curva de enfriamiento de la aleación del cuadro 1, así como la curva de derivada correspondiente $\left(\mathrm{en}^{\circ} \mathrm{C} / \mathrm{s}\right)$. Se observa que cuando la derivada es cero comienza la solidificación de Al. Según los datos registrados esta temperatura de fusión es de $659.49^{\circ} \mathrm{C}$. Si tomamos en cuenta que el valor real o típico es de $660^{\circ} \mathrm{C}$ (Backerud et al., 1990), entonces resulta un error relativo del 0.08\%. aunque es un error tan pequeño hasta ahora, el algoritmo del programa de mínimos cuadrados ha dado buenos resultados. 


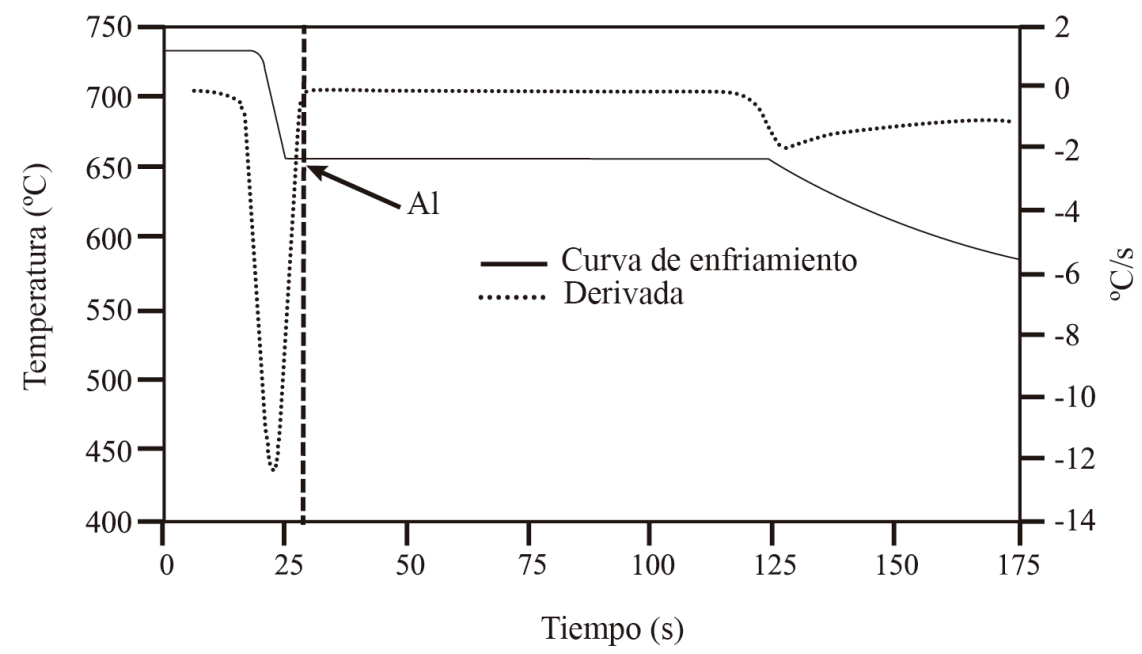

GRÁFICA 1

Curva de enfriamiento y derivada de una aleación Al-0.13\%Fe- $0.05 \% \mathrm{Si}$ Fuente: elaboración propia.

Cabe mencionar que por ser un sistema experimental se tienen errores de medición por termopares, por sensores, por perturbaciones electromagnéticas externas hacia la tarjeta de adquisición de datos. Todos estos errores contribuyeron al error total en la medición del punto de fusión de $659.49^{\circ} \mathrm{C}$ con su error relativo del $0.08 \%$.

Ahora se pueden hacer las siguientes preguntas: ¿el cálculo de derivada utilizando el algoritmo del programa de mínimos cuadrados tiene un error significativo con la derivada real? ¿Este error de derivada a su vez puede incrementar todavía más el error en el cálculo de la temperatura de fusión con la curva de enfriamiento con la temperatura de fusión real? También se podría decir que puede haber una compensación del error al calcular la derivada con los errores de medición mencionados al calcular la temperatura de fusión y con esto tener un error aparentemente pequeño de la temperatura de fusión. Todavía se puede ir más lejos en el análisis y preguntarse si el programa de mínimos cuadrados funciona para cualquier aleación y en cualquier condición de enfriamiento. Para resolver estos problemas es necesario poner a prueba el algoritmo del programa de mínimos cuadrados con una función ya conocida y afrontar los resultados.

En la gráfica 2 se muestra la gráfica de la función $f(x)=$ senx y la curva de su derivada utilizando el programa de mínimos cuadrados. Los datos se graficaron utilizando el programa SigmaPlot ${ }^{\circ}$ y se utilizó una tabulación de 201 datos en un rango de 0 a 4 para los valores de $x$. 


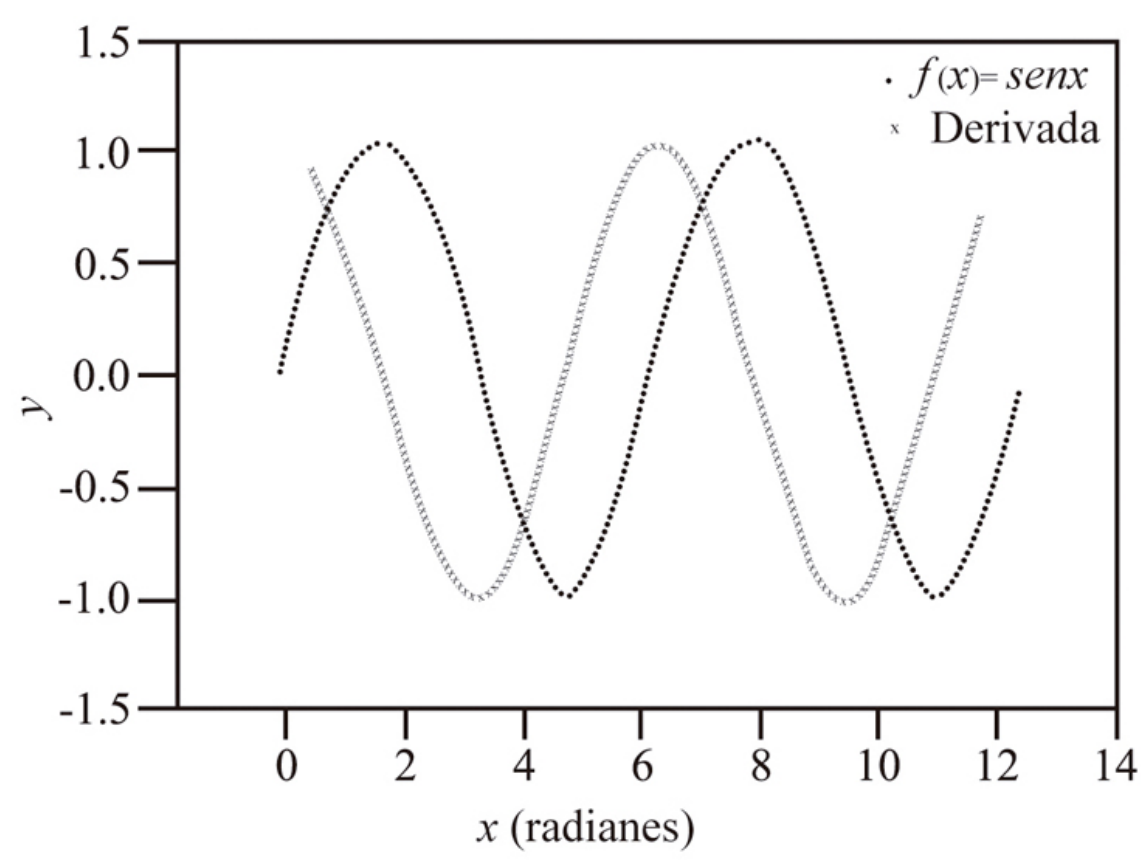

\section{GRÁFICA 2}

Función $f(x)=\operatorname{sen} x$ y su derivada utilizando el programa de mínimos cuadrados

Fuente: elaboración propia

En la gráfica 3 se muestra nuevamente la curva de derivada obtenida del programa de mínimos cuadrados, y también la gráfica de $g(x)=\cos x$. En esta figura se observa que las dos curvas tienen una aproximación bastante buena. Se realizó una prueba de correlación de Pearson con el mismo programa SigmaPlot ${ }^{\circledR}$ y dio como resultado un coeficiente de correlación de 1.0, lo que significa que prácticamente es la misma curva. Se trataba de llegar a la conclusión anterior si se toma en cuenta que la derivada de la función senx es el $\cos x$ (Leithold, 2014). 


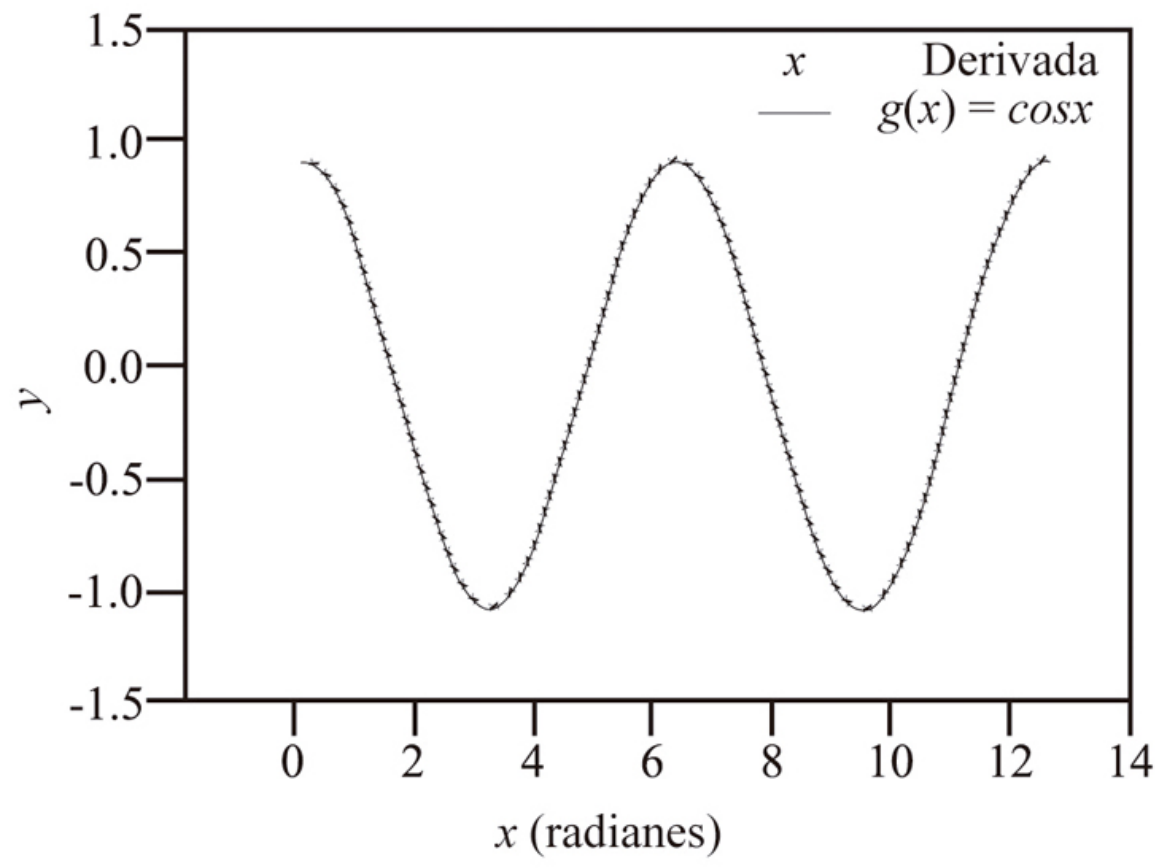

GRÁFICA 3

Derivada de la función sen $x$ obtenida con el programa de mínimos cuadrados y la función $g(x)=\cos x$ Fuente: elaboración propia.

Puesto que el algoritmo del programa de mínimos cuadrados dio buen resultado con las funciones senx y $\cos x$, se puede también tomar en cuenta que cualquier función periódica y continua a trozos se puede aproximar a una serie de Furier (Butkov, 1973) y, por tanto, se podría utilizar con una buena aproximación la derivada de estas funciones con el programa de mínimos cuadrados.

Un ejemplo práctico es el siguiente: si a un estudiante de licenciatura se le pide encontrar la derivada de la función $Q(x)=\tan x$, entonces podría utilizar el programa de mínimos cuadrados y orientarse gráficamente antes de proceder a encontrar el resultado de manera analítica. La gráfica 4 muestra la función $Q(x)=\tan x$ y para poder hacerla se utilizaron 1600 datos para el valor de $x$ en un rango de $-2 \pi$ a $2 \pi$. 


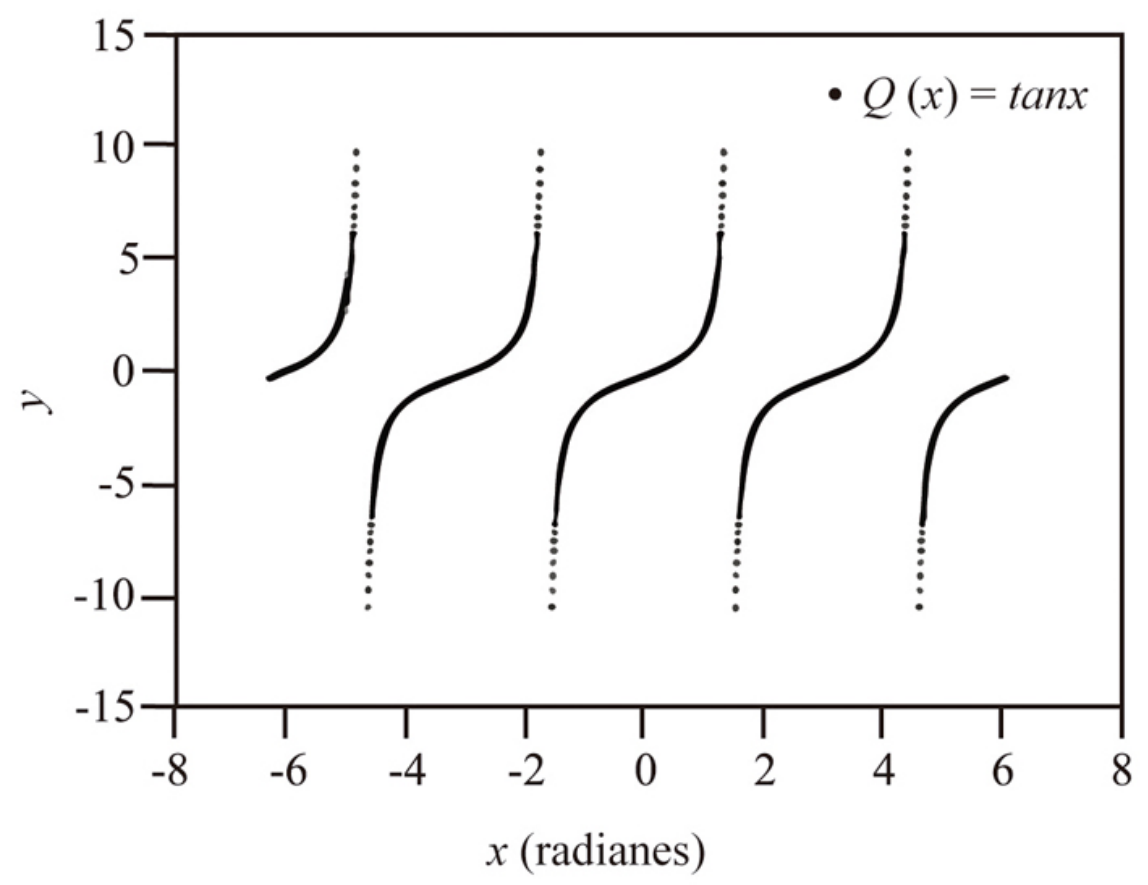

GRÁFICA 4

Gráfica de la función $Q(x)=\tan x$

Fuente: elaboración propia.

La gráfica 5 muestra el resultado de la derivada de la función $Q(x)=\tan x$ utilizando el programa de mínimos cuadrados y la gráfica de la función $h(x)=\sec ^{2} x$. Como es de esperarse, las gráficas coinciden, puesto que es bien sabido que la derivada de la $\tan x$ es la $\sec ^{2} x$ (Leithold, 2014). Nuevamente se realizó la prueba de correlación de Pearson con el programa SigmaPlot ${ }^{\circ}$ y dio como resultado un coeficiente de correlación de 1.0. Con esto se prueba que, aunque la función sea difícil de graficar el programa de mínimos cuadrados, sigue arrojando buenos resultados. 


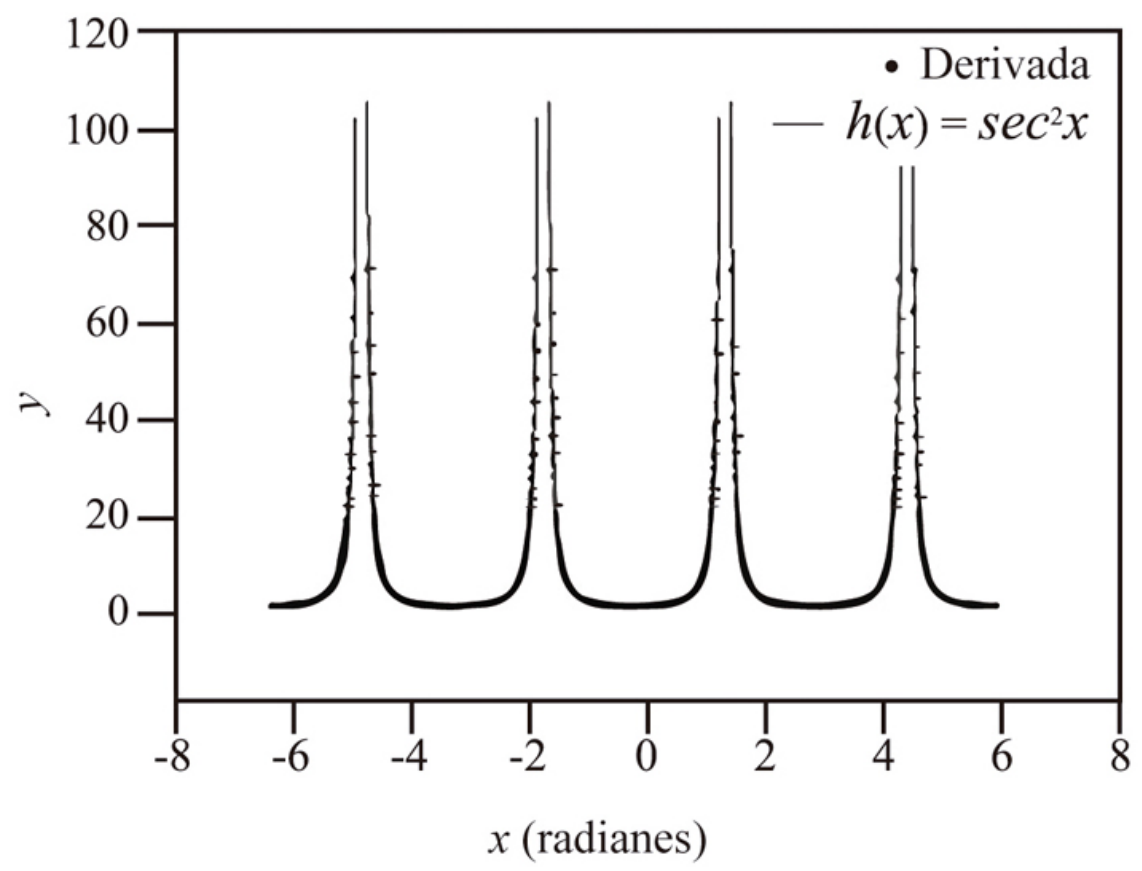

\section{GRÁFICA 5}

Derivada de la función $\tan x$ obtenida con el programa de mínimos cuadrados y la función $h(x)=\sec ^{2} x$ Fuente: elaboración propia.

\section{Prospectiva}

El algoritmo del programa de mínimos cuadrados dio buenos resultados con la aleación estudiada, con velocidades de enfriamiento tan altas como $12{ }^{\circ} \mathrm{C} / \mathrm{s}$. Entonces, en un futuro este algoritmo se podría utilizar fácilmente para cualquier otra aleación con velocidades de enfriamiento más bajas en las cuales se obtendría un análisis térmico todavía más certero. El algoritmo también dio buenos resultados en curvas teóricas como la función tangente, por lo que en un futuro próximo podría ser una herramienta para estudiantes de cálculo diferencial junto con sus libros. También, se podría implementar el código en cualquier otro programa y dar pie a ser material para desarrolladores, inclusive el algoritmo podría ejecutarse desde un dispositivo más portable como un teléfono celular.

\section{Conclusiones}

En este trabajo la aplicación numérica de la derivada, basada en el método de mínimos cuadrados, dio como resultado el análisis térmico de una aleación de aluminio y se deja la posibilidad de analizar de esta forma cualquier otra aleación. Los resultados muestran una buena aproximación experimental de la temperatura de fusión de $659.49^{\circ} \mathrm{C}$ de la aleación de aluminio que comparada con la temperatura de fusión real del aluminio de $660^{\circ} \mathrm{C}$ da como resultado un error relativo de $0.08 \%$.

Se puso también a prueba el algoritmo del programa de mínimos cuadrados con las funciones senx y $\cos x$ dando buenos resultados, por lo que se puede asumir que cualquier función periódica y continua a trozos se puede aproximar a una serie de Furier y de esta forma se podría utilizar con una buena aproximación la derivada de estas funciones con el programa de mínimos cuadrados. 
Por último, se puso a prueba el programa de mínimos cuadrados derivando una función difícil de graficar como lo es la $\tan x$ y su derivada la $\sec ^{2} x$. Para todas las aproximaciones de las derivadas obtenidas con el programa de mínimos cuadrados comparadas con las funciones reales dieron como resultado un coeficiente de correlación de 1.0, que muestra así el potencial de este programa, sobre todo para ser utilizado por estudiantes de licenciatura en cálculo diferencial e investigadores que requieran de la derivada con análisis numérico.

\section{REFERENCIAS}

Backerud, L., Chai, G. y Tamminen, J. (1990). Solidification characteristic of aluminum alloys. Volume 2. Oslo AFS/ SkanAluminum.

Baird, D. C. (1991). Experimentación. Una introducción a la teoría de mediciones y al diseño de experimentos. Segunda Edición. México: Prentice-Hall.

Biacino, L. (2014). A geometrical solution by Fermat to a problem of maximum. Applied Mathematical Science, 8(136), 6827-6834.

Butkov, E. (1973). Mathematical Physics. New York: Addison Wesley.

Chapra, S. C. y Canale, R. P. (2007). Métodos numéricos para ingenieros. Quinta edición. México: McGraw-Hill.

Fermat, P. (1891 y 1912). Methodus ad disquirendam maximam et minimam, V. Ad Methodum de maxima et minima Appendix. Paris: Gauthiers-Villars.

Leithold, L. (2014). El cálculo. Séptima edición. Mexico: Oxford University Press.

Martínez, J. A., Valenzuela, J., Hernández, M. P. y Herrera, J. (2016). Automatización de un microscopio de barrido por efecto túnel utilizando una tarjeta OMB-DaqBoard/2000 y Labview. Revista Mexicana de Física, 62, 45-50.

Narayanan, L. A., Samuel, F. H. y Gruzleski, J. E. (1994). Crystallization behavior of iron-containing intermetallic compounds in 319 aluminum alloy. Metallurgical and Materials Transactions A. (25A), 1761-1773.

Singh, K., Sharma, A., Rajput, G. y Kasturia, D. (2014). Application of derivatives in real life. International Journal of Research, 1, 337-339.

\section{ANEXO}

\section{Algoritmo del programa de minimos cuadrados en código de SigmaPlot ${ }^{\circ}$}

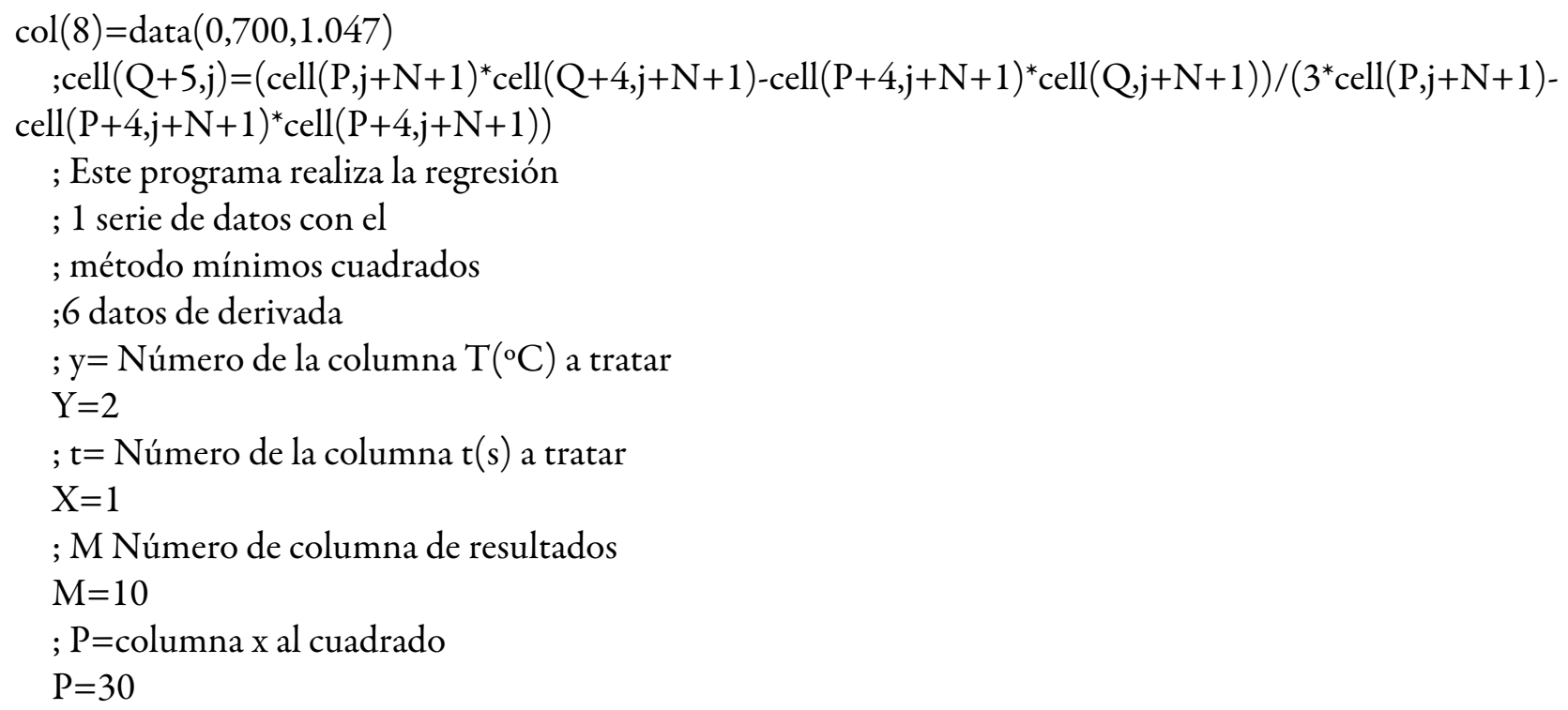




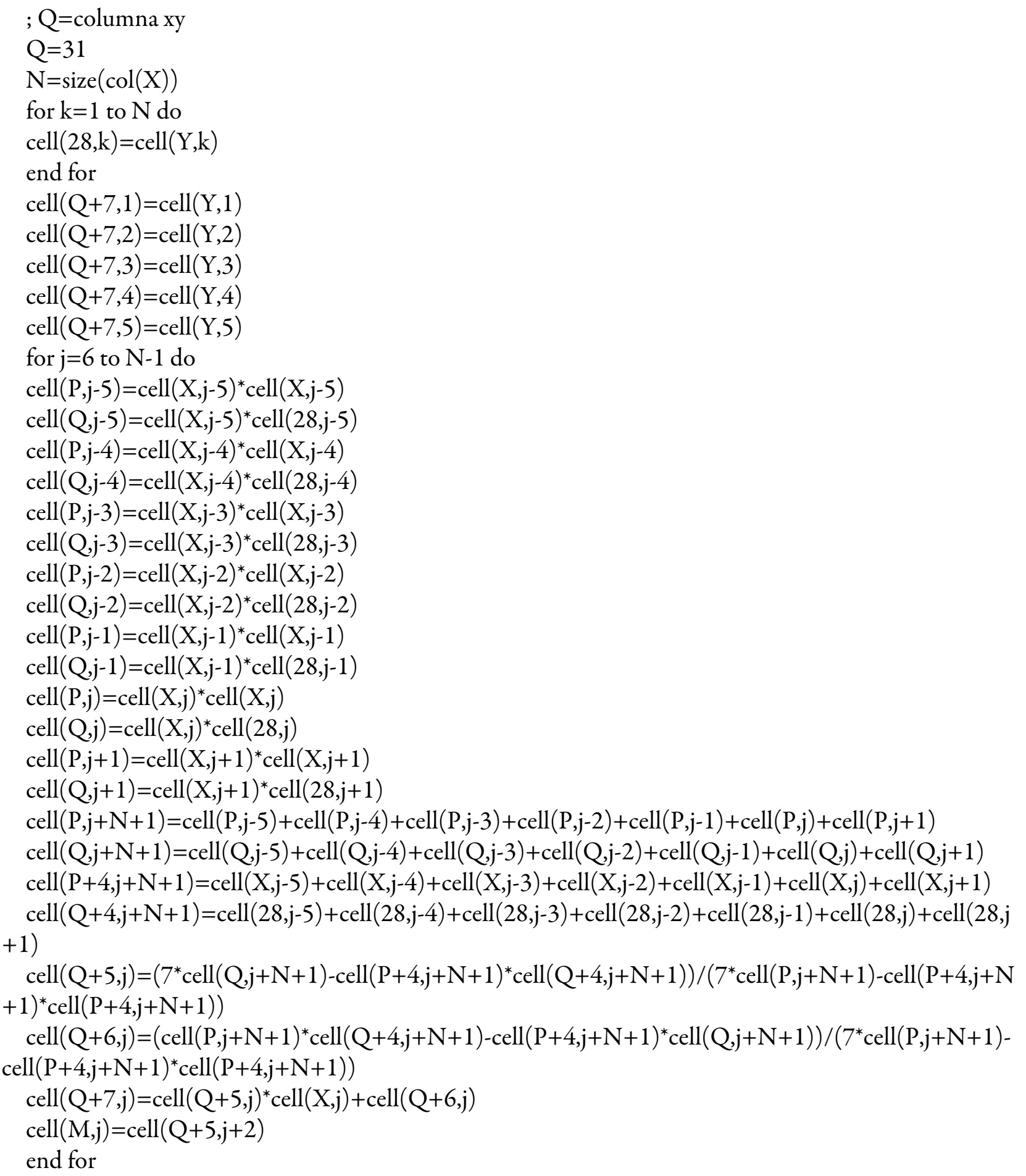

\section{BY-NC-ND}

